\title{
Using geographical information systems for defining the accessibility to health care facilities in Jeddah City, Saudi Arabia
}

\author{
Abdulkader A. Murad ${ }^{1}$ \\ ${ }^{1}$ Department of Urban and Regional Planning, Faculty of Environmental Design, King Abdulaziz University, \\ Jeddah, Saudi Arabia
}

\begin{abstract}
Spatial data play an important role in the planning of health care facilities and their allocation. Today, geographical information systems (GIS) provide useful techniques for capturing, maintaining and analysing health care spatial data; indeed health geoinformatics is an emerging discipline that uses innovative geospatial technology to investigate health issues. The purpose of this paper is to define how GIS can be used for assessing the level of accessibility to health care. The paper identifies the advantages of using GIS in health care planning and covers GIS-based international accessibility with a focus on GIS applications for health care facilities in Jeddah city, Saudi Arabia. A geodatabase that includes location of health services, road networks, health care demand and population districts was created using ArcGIS software. The geodatabase produced is based on collected data and covers issues, such as defining the spatial distribution of health care facilities, evaluating health demand types and modelling health service areas based on analysis of driving-time and straight-line distances.
\end{abstract}

Keywords: geographical information systems, health accessibility, network analysis, health facilities, Saudi Arabia.

\section{Introduction}

Access to health care has long been of concern to community and public health planners. Previous research on this issue has focused on two distinct and usually non-overlapping areas. One considers the spatial dimensions related to geographic access (distances, travel times, catchments, etc.), with data being manipulated and geographically analysed using geographical information systems (GIS) before subsequent statistical analyses; the other examines service accessibility by considering the socioeconomic aspects of access related to cost, insurance provision etc., with data collected using opinion or attitude surveys. In both cases, the overriding objective is to inform spatial planning and health policy making (Comber et al., 2011). Researchers have defined access from various aspects and interpreted it as two separate components: economic and physical access. The former is the ability to purchase health care services, whereas

\footnotetext{
Corresponding author:

Abdulkader A. Murad

Department of Urban and Regional Planning

Faculty of Environmental Design, King Abdulaziz University

P.O. Box 80210, Jeddah 21589, Saudi Arabia

Tel. +966 2 640-200; Fax +966 2 640-1328

E-mail: amurad@kau.edu.sa
}

the latter is the ability to overcome the cost of distance (Cheng et al., 2012). In addition, health access can be divided into four components: potential access (enabling resources), realised access (actual use of health service), equitable access (occurring when resources meet the needs of the population with various demographic characteristics) and inequitable access (occurring when the prevailing social structure, health beliefs, and enabling resources determine who gets medical care).

The simplest definition of accessibility of a given location is to describe it in terms of how easy it is to get there. When talking about something being accessible in colloquial language, one generally uses "gettable" as pointed out by Moseley (1979). However, the reasons why someone or something may be inaccessible (or difficult to get at) may vary considerably. The reason for such variety can result from the concepts of physical and social dimensions of accessibility. The former refers to the ability to command the transportation network facilities needed for reaching supply locations at suitable times. The social dimension, on the other hand, focuses on the individuals meeting certain requirements in terms of age, or ability to pay, in order to be permitted to pass the symbolic barrier around the supply location that needs to be reached. Accordingly, what is accessible by private car may be inaccessible by 
public transport, and what is within accessible walking distance for the young may be effectively inaccessible for senior citizens (Jons and Van Eck, 1990). Another definition of accessibility that emphasises accessibility as consisting of four dimensions (each of which has a supply and demand element) has been proposed by Munoz and Källestål (2012). These four dimensions are:

(i) Geographical accessibility - the physical distance or travel time between the service delivery point and the user;

(ii) Availability - the opportunity to access the right type of health care services when needed as well as having the appropriate type of service providers, materials and equipment;

(iii) Financial accessibility - the relationship between the price of services and the willingness and ability of users to pay for those services, as well as protection from the financial consequences of health expenses; and

(iv) Acceptability - the responsiveness of health service providers to the social and cultural expectations of individual users and communities.

The spatial or geographic aspects of health provision and access to facilities have been considered in previous research. The straight-line or road distance to health services are typically measured and analysed in order to quantify differences in access and gaps in service provision to model optimal facility location and identify inequalities in service provision. Recent examples of these purely spatial approaches in health science include identifying health catchments, examining equity of access for different social groups and modelling spatial patterns of facility usage and access.

According to Phillips (1990), health care accessibility can be divided into "revealed" and "potential" accessibility. The former focuses on the actual use of health care services, while the latter is concerned with the geographical patterns and aggregate supply of medical care resources (Thouez et al., 1988). These two categories can be further divided into spatial and non-spatial accessibility as proposed by Luo (2004). Spatial accessibility emphasises the importance of geographic barriers (distance or time) between consumer and provider, whereas non-spatial accessibility focuses on non-geographic barriers or facilitators, such as social class, income, ethnicity, age, sex, etc. (Wang and Luo, 2004).

As seen in the literature, there is a need for quantitative indicators of accessibility to different kinds of public health services that could serve as instruments in the comparisons of accessibility in different parts of the region and in the evaluation of alternative plans for new service facilities and transportation links (AlSahili et al., 1992). Examples of accessibility indicators include provider-to-population ratios, distance to nearest provider, average distance to a set of providers and gravitational models of provider influence (Guagliardo et al., 2003). Each of these indicators can be used to evaluate accessibility to health care centres. For example, Luo (2003) has determined accessibility to physician locations west of Chicago based on the physician to population ratio. The same principle has been applied by Wang and Luo (2004) but with adding non-spatial factors to health care accessibility such as age and social class. Murad (2004) have presented the use of gravity models in defining the flow of population to health centres in Saudi Arabia, and the same technique is also found in the work by Birkin et al. (1996) for defining patient flows to health centres in the UK. The gravity-based models produce a combined accessibility indicator of distance and availability and can provide the most valid measures of spatial accessibility (Guagliado et al., 2003).

GIS has several spatial analysis tools that can be applied to solve planning problems. These applications vary from land use analysis to facility planning and optimisation studies and can be applied in the health sector by creating shapefiles of health centres including various types of health services supply data. One of the important issues in this type of planning is to define the accessibility of to health care facilities in any built-up area. GIS can be of great help for studies that need to define the level of accessibility at certain location. The tools available are either related to raster-based maps or to vector data. The former represents data as cell matrices in continuous space, while the latter defines features such as points, lines and polygons. The vector approach is generally more useful for the purposes discussed here. For example, GIS proximity analysis, commonly known as buffer operation, is based on the distance derived from certain selected features. The output of this type of analysis can be used to evaluate the accessibility of any location according to the factor of distance. Most GIS software applications offer this kind of distance-based analysis, for example to be used for identifying risk areas by defining zones or buffers around features like rivers to denote risk of flooding (Grimshaw, 2000). Advanced tools for defining accessibility of vector data are available in ArcGIS software (ESRI; Redlands, CA, USA) that includes gravity models or 
spatial interaction models for testing and evaluation of the likely trade areas defined by iso-probability contours. In this paper, we emphasise the spatial aspects and the techniques for measuring health care accessibility using an updated approach of spatial accessibility measures, such as GIS-based straightline distance and GIS-based drive time.

Visualisation of spatial data is the first step in obtaining an initial overview of data distribution and possible spatial patterns as well as a component of the broader approach of exploratory spatial data analysis (ESDA). Here several techniques can be used based either on the vector or the raster presentation. The four main GIS classification methods are the following:

(i) The natural-breaks method, which groups data based on the natural distribution of their values and sets class breaks where there is a jump in value. GIS determines the high and low value of each class using a mathematical procedure. The software chooses the breaks that best group similar values to maximise the differences between classes with uneven value distributions.

(ii) The quintile method, which divide data into classes containing equal numbers of features. The features are ordered based on attribute value summing the number of features as it goes. However, quintiles can mislead in areas of greatly varying size.

(iii) The equal-interval method. Here the difference between high and low values is the same for every class. The lowest value is subtracted from the highest and then divided by the number of specified classes.

(iv) The standard deviation (SD) method, which places feature classes based on how much their values vary from the mean in order to identify which features are above or below the average value. The mean and the standard deviation are calculated by the software that subsequently adds or subtracts the standard deviation to or from the mean to set the data class breaks.

If health data are unevenly distributed, the naturalbreaks method is suitable. However, if the data are evenly distributed and the relative difference between features has to be emphasised, the quintile method is appropriate. Data classification can be manually defined in GIS, which allows for class breaks of clinical significance in a health setting. For example, if a known feature of a given disease has some economic impact or service demand requirement, then this number can be set as a class break.

The ArcGIS Network Analyst provides network- based spatial analysis, such as routing, fleet routing, travel directions, closest facility, service area and location-allocation, where the user can dynamically model network conditions, including one-way streets, turn and height restrictions, speed limits and variable travel speeds based on the prevailing traffic. Apart from finding the shortest routes, locating the closest health centres and create networks using existing GIS data, the user can also

(i) produce the most efficient routes for a fleet of vehicles that visit many locations;

(ii) decide when vehicles can arrive at locations by using time windows;

(iii) determine optimal locations for facilities by performing location-allocation analysis;

(iv) define service areas based on travel time or distance; and

(v) generate matrices of network travel costs from each origin to any destination.

Several methods that can be used for defining the health service area. For example, Roovali and Kilvet (2006) used the travel-time method for defining geographical variation in hospital use in Estonia, while Jordan et al. (2004) defined health service areas in Southwest England by using both travel-time and straight-line techniques. Identifying the relationship between supply and demand/utilisation is important for health care planners. For example, Murad (2008) presented a GIS application for modelling health catchment areas using the straight-line distance to the closest source of health care, while Lee and Moon (2014) analysed location strategies, an approach that should be useful for decision-makers concerned with the distribution of hospitals and other health care centres. Grid models were used to assess urban hospital distribution in Seoul, South Korea. Another recent example of using GIS for health accessibly application has been provided by Ranga and Panda (2014), who used a three-step floating catchment area (3SFCA) method, a modification of the simple gravity model, to examine spatial access to in-patient health care in northern rural India. Lwasa (2007) used GIS for analysis of access to health facilities applying the population gridding approach, coupled with location of health infrastructure facilities for decision support in health services planning. These studies conclude that distance to the location of the closest health facility is an important factor when evaluating health service areas. Against this background, we investigted access to health care in a particular place, Jeddah City, Saudi Arabia. 


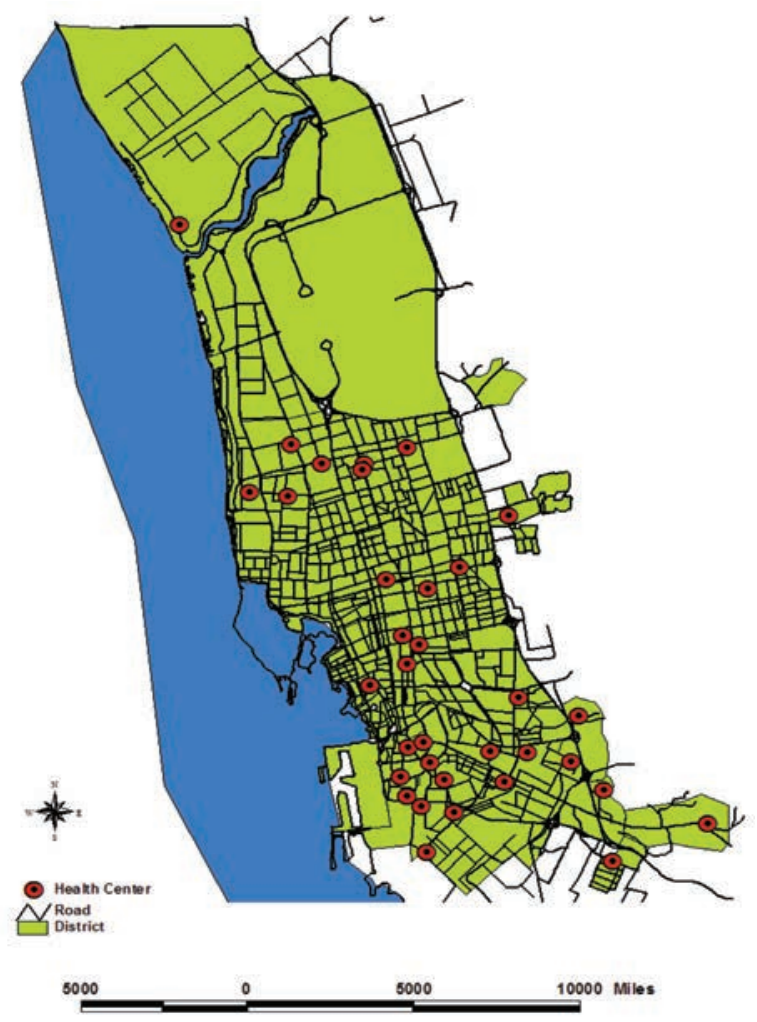

Fig. 1. Spatial distribution of public health centres at Jeddah City.

\section{Materials and methods}

The study area was Jeddah City (Fig. 1), where different types of health care facilities - governmental and private health-centre general or specialised hospitals are available for the residents. These centres face different challenges such as matching the available resources with the expressed demand. The ArcGISArcInfo and ArcGIS-Arcview (Version 10.1) software packages were used for health data visualisation, spatial analysis, and modeling. All health centres were plotted on a GIS-generated map as point features and classified based on staff, supplies or service. The different types of staff considered were the following:

(i) physicians (general practitioners);

(ii) family physicians;

(iii) dentists;

(iv) nurses;

(v) midwives;

(vi) pharmacists (pharmacologists);

(vii) laboratory technicians;

(viii) X-ray technicians;

(ix) administrators;

(x) general service staff; and

(xi) staff with miscellenous responsibilities.
In order to build the pre-defined accessibility models, several datasets were collected and captured into the ArcGIS software. The data were non-digital, so transforming them into digital format was a major activity in the present study. First, the road network for Jeddah City was created as ArcGIS Coverage that describes the pattern of road network and includes data about road length and road types. Point GIS Coverage was used to define the location of all local health centers in Jeddah city. It included several attributes such as size of the facilities, area, registered patient information, equipment available, etc. This was done since the coverage can be classified based on any of these attributes and the results can be used for comparing health centers. Fig. 1 describes the spatial differences from the point of view of how many doctors are available per area. The third important piece of information for this study was delineation of the boundaries of the health centres. It took the form of polygon coverage thet divided the city into several zones where each zone shows the spatial extent of its health facility. Several attributes were linked to this coverage including name of the health centre, population size, patient size, and the extent of the area covered.

GIS was also applied for multiple-data classification, i.e. logical operations that deal with the database through multiple queries allowing the user to identify and select features by a special sets of criteria with more than one parameter. For example, one could find health centres with many physicians but with fewer nurses. The features were identified and selected from the database and highlighted on the map according to a combination of several conditions and saved in a new Coverage file for further analysis. Defining health accessibility is a major task in any local health care planning. To obtain this type of analysis, GIS functions on vector or raster datasets were used. A straight-line distance function was applied on health centre shapefiles. The presented application produced a health accessibility model based on the approach of nearest unction that created a distance surface with a continuous distance from a source based on straight line (Euclidean) distance using simple geometry based on the following formula:

$$
\text { Distance }=\sqrt{\left(x^{1}-x^{2}\right)^{2}+\left(y^{1}-y^{2}\right)^{2}}
$$

Each cell in the created distance surface has a potentially unique value. The output of this function can be displayed using graded colours, either as a continuous 


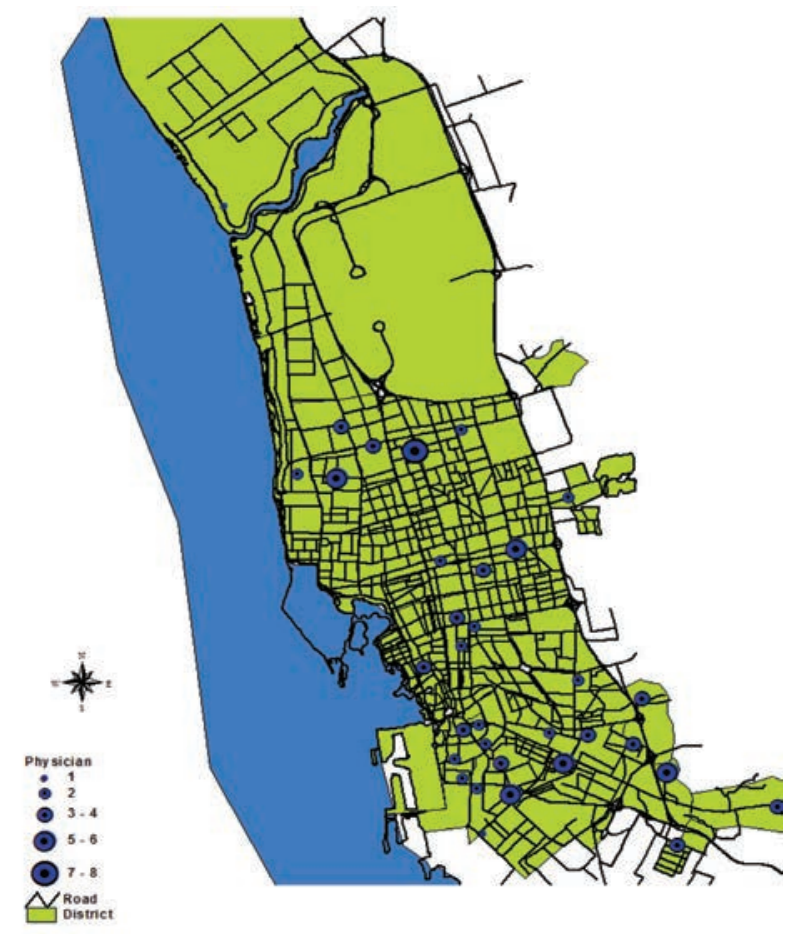

5000

10000 Miles

Fig. 2. Classification of public health centres based on the number of physicians.

range or grouped into classes for the purpose of analysing the resulting patterns. The ArcGIS Spatial Analyst provides several distance mapping tools for measuring straight-line (Euclidean) distance and distance measured in terms of other factors such as slope, current road infrastructure and land use. The calculation of the accumulated cost of traveling, or mapping distance, provides the user with additional data, which are useful for the final decision. For example, the accumulated least cost of travel to a number of health centres can be calculated, taking into consideration any potential travel obstacles.

Euclidean distance and cost distance are the two main ways to perform distance analysis in ArcGIS Spatial Analyst. The Euclidean distance functions measure the straight-line distance from each cell to the closest source. not only determining allocation, but also calculating the distance and direction to the closest source.

\section{Results}

Each health centre has a different number of physicians and/or nurses. For example, the Alsafa Centre and the Gulail Centre engage more physicians than the

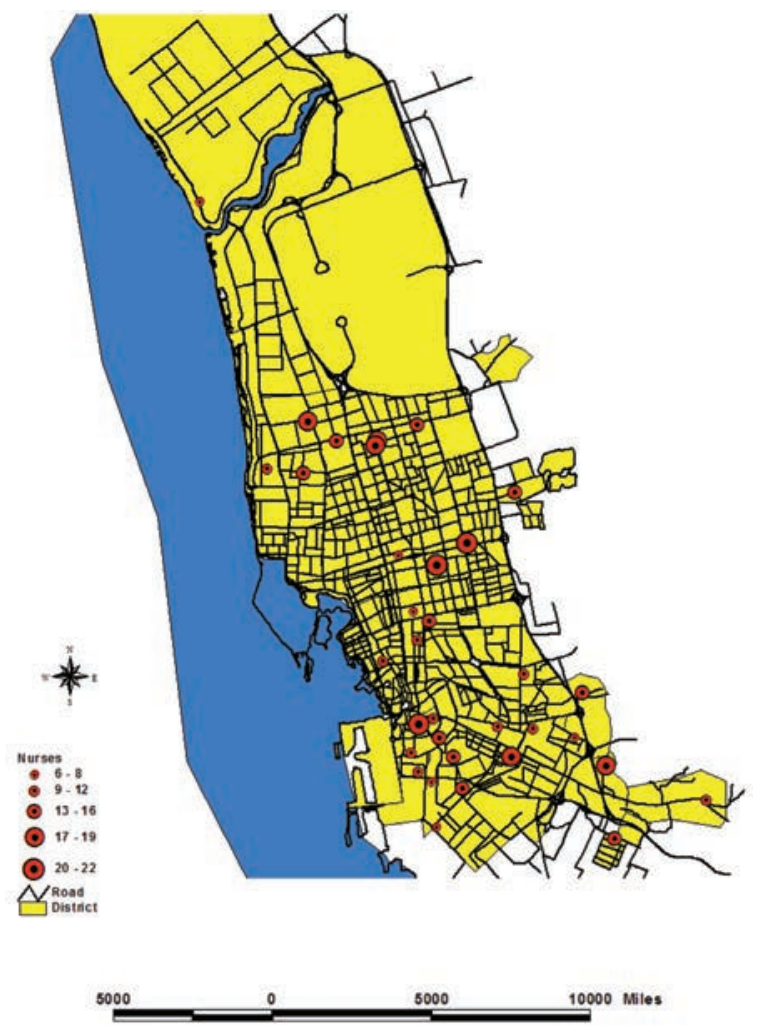

Fig. 3. Classification of public health centres based on the number of nurses.
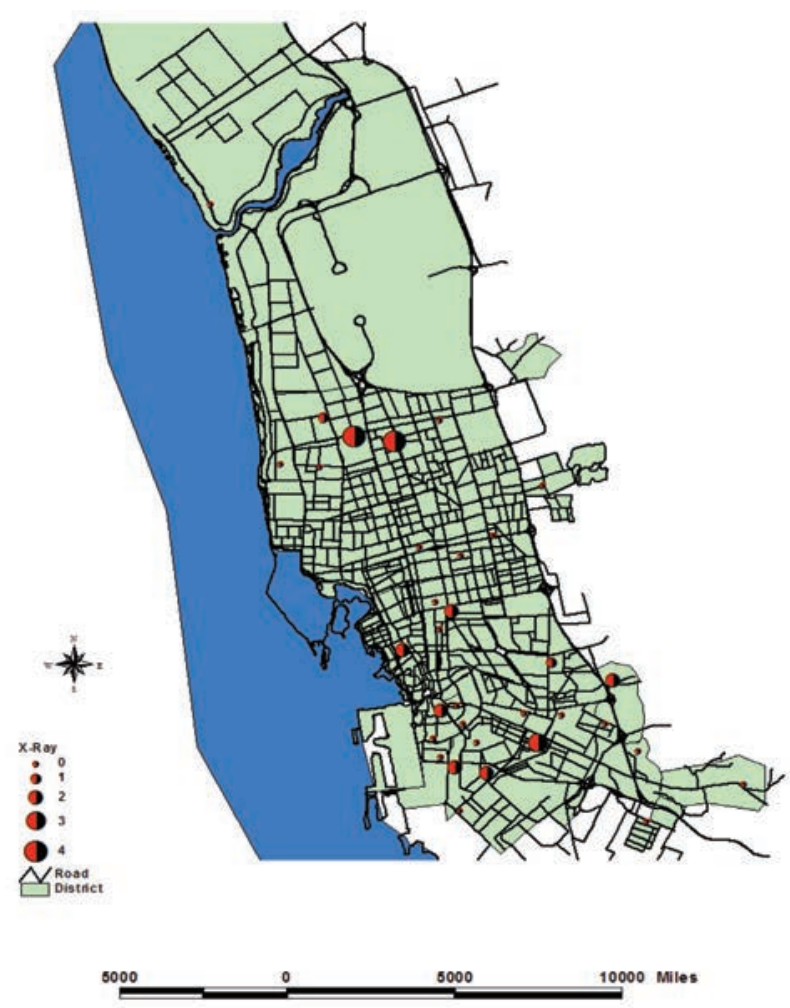

Fig. 4. Classification of public health centres based on the number of available staff for X-ray investigations. 
Alsharafia Centre and the Althayuar Centre. One reason for this difference is related to the size of existing demand. Fig. 2 shows a classification model for a health centre based on the number of physicians, while Fig. 3 demonstrates health centres based on the number of nurses and Fig. 4 shows health centres based on $\mathrm{X}$-ray staff. Each figure provides a visual evaluation and comparison between health centres based on their existing supply. Large areas with low population rates can be more attractive than smaller areas receiving larger populations. To overcome this problem, GIS can be used for visualising eatures in proportions to their relative health data. Accordingly, features that have large populations are presented with larger symbols, while low low populations are shown with smaller icons.

The presented application has produced two types of health accessibility models the drive-time technique and the straight-line distance technique. The following part of the paper illustrates the outputs of these GISbased techniques by showing a service area based on drive-time for health services located in Jeddah. Travel time is calculated for all Jeddah roads and used for producing a 5-min drive-time area. ArcGIS software was used to select all parts of the city that fall within the 5 -min service area of health services. These districts form the main demand parts for the health services and should be studied and monitored by health planners for the purpose of health care marketing and utilisation. Fig. 6 presents the visualized health service area and shows that districts like Alzahra and Alnahda are not well served presently.

This type of analysis results in two main conclusions. The first one is related to identifying health served areas which fall inside the driving time catchment areas. The second regards the identification of un-service areas, of the city that should be evaluated by health planners. Based on the output of this type of analysis, only $30 \%$ of Jeddah City falls within the resulting health catchment area. Meanwhile, $70 \%$ of the city residents have poor accessibility to their nearest health centre. Several districts located north of Jeddah are not well served by health centres. This means that residents north of Jeddah must travel more than $5 \mathrm{~min}$ in order to reach to their nearest health centre. Accordingly, the local health authority of Jeddah City should make plans to introduce additional health centres in these un-served areas.

Fig. 7 displays the results of health care distance surface with values ranking from 1 to 12 where, $1=$ very accessible location and $12=$ least accessible location. One of the main findings of this model is that large

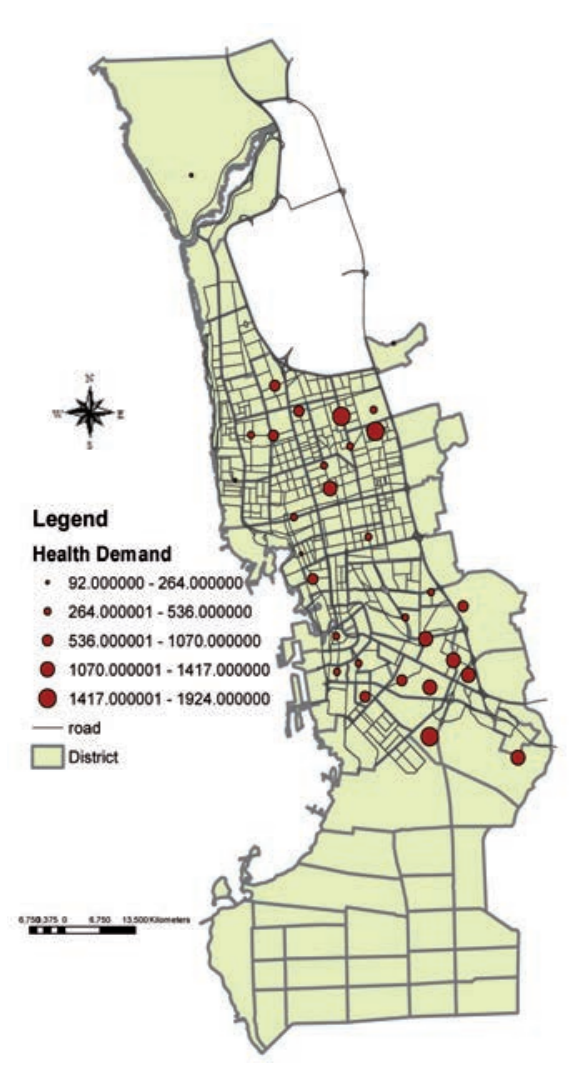

Fig. 5. Classification of public health centres based on the number of patients.

parts of Jeddah City, mainly in the northern districts have low accessibility scores, which means that residents there need to travel longer distances in order to reach their nearest health care centre. The same modelling technique was used with the road network data feature to define city parts that have good accessibility as well as the parts where it is poor (Fig. 7). This output was further used in the presented application for

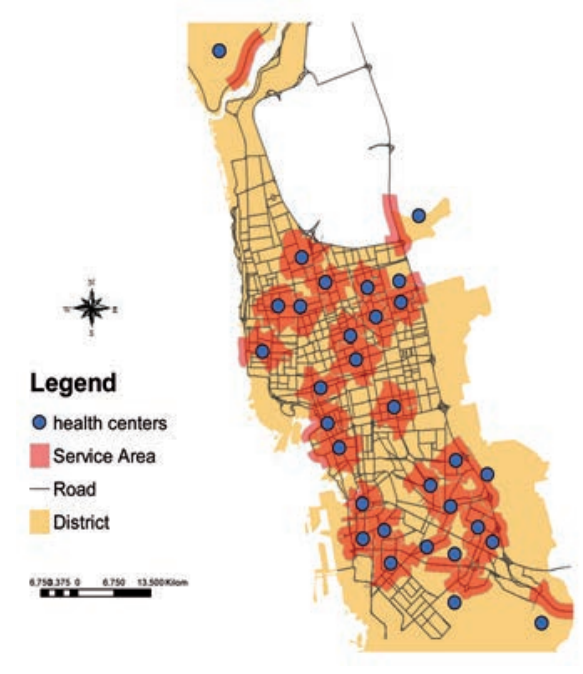

Fig. 6. Service areas located within 5-min drive-time. 

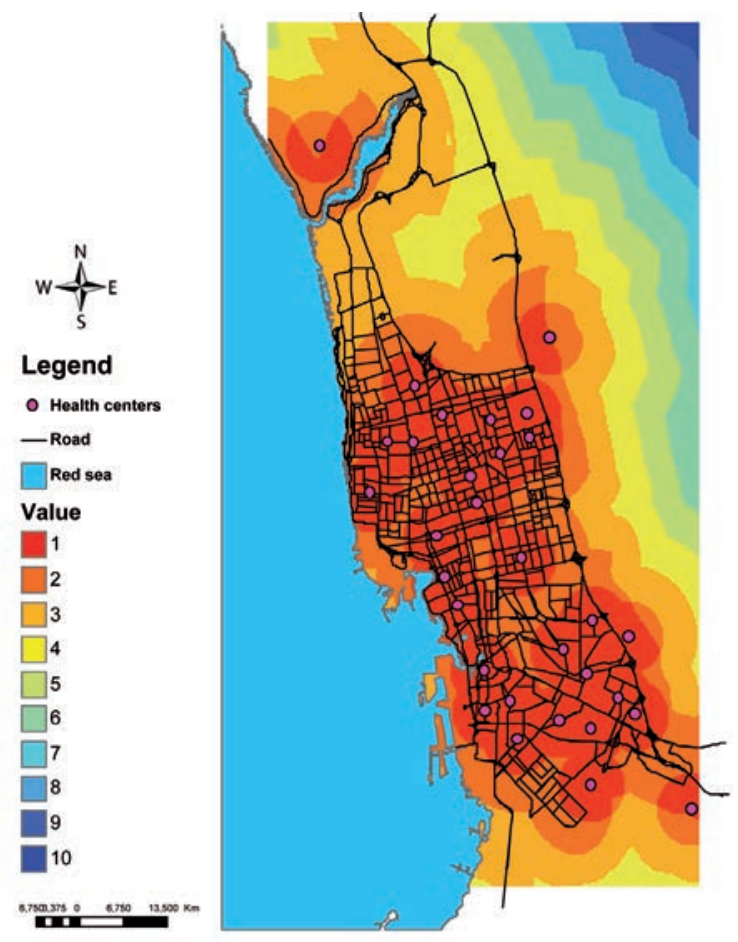

Fig. 7. Accessibility to public health care centres in Jeddah City (based on distance). Location varies from $1=$ very accessible location and $10=$ least accessible location.

the purpose of producing accessibility surface that takes in to account distance to health centres and distance to road networks. ArcGIS map algebra technique was used to produce the accumulative accessibility model (Fig. 8). The results of this model help health planners in Jeddah City to identify areas with poor accessibility as a result of distance from health centres and from road networks. Several parts of the city are located in poor accessibility zones, particularly in the North and in the East. Accordingly, more health care centres should be allocated to these locations.

\section{Discussion}

Although distance is a fundamental indicator of geographical access, travel time, cost, transportation access and perceived distance are often much more relevant to health care utilisation. Using GIS, health planners can estimate travel time along road networks, taking into account average speeds and speed limits on different classes of roads and physical barriers. Travel time provides a better indication of geographical barriers to health services than does travel distance since, by definition, travel times incorporate access to transportation. The mode of transportation is also important in estimating travel time. For example, to evaluate accessibility by bus, researchers have focused on the frequency of

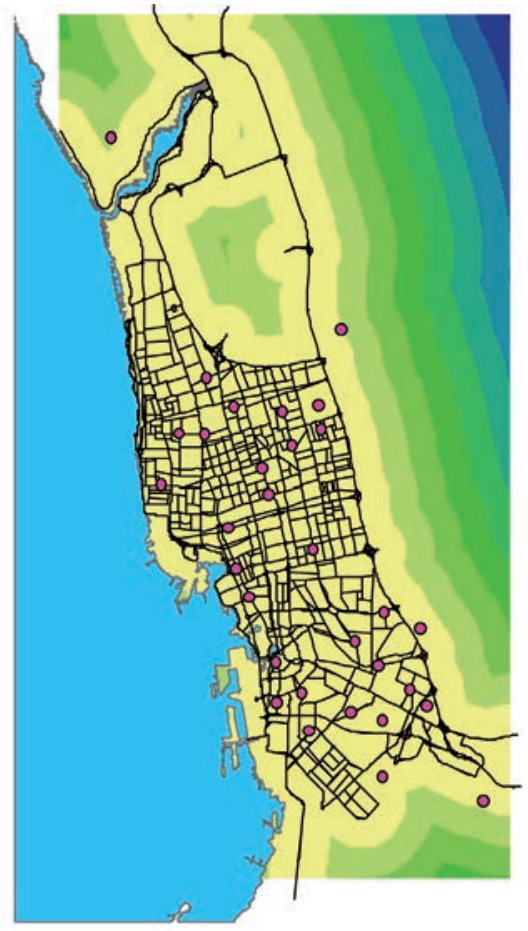

Fig. 8. Accessibility to main roads in Jeddah City varies from very accessible (1) to least accessible (10).

bus services and whether or not residents could walk to a bus stop. Walking and use of public transportation are especially important in developing countries where

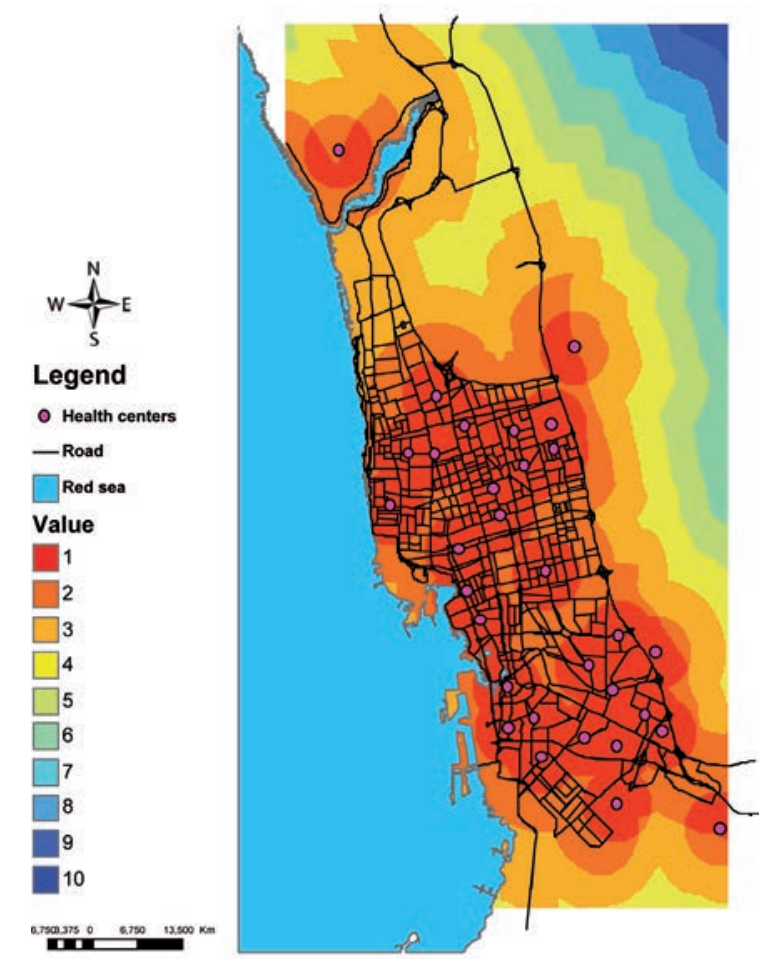

Fig. 9. Proximity model for public health centers varies from very accessible (1) to least accessible (10). 


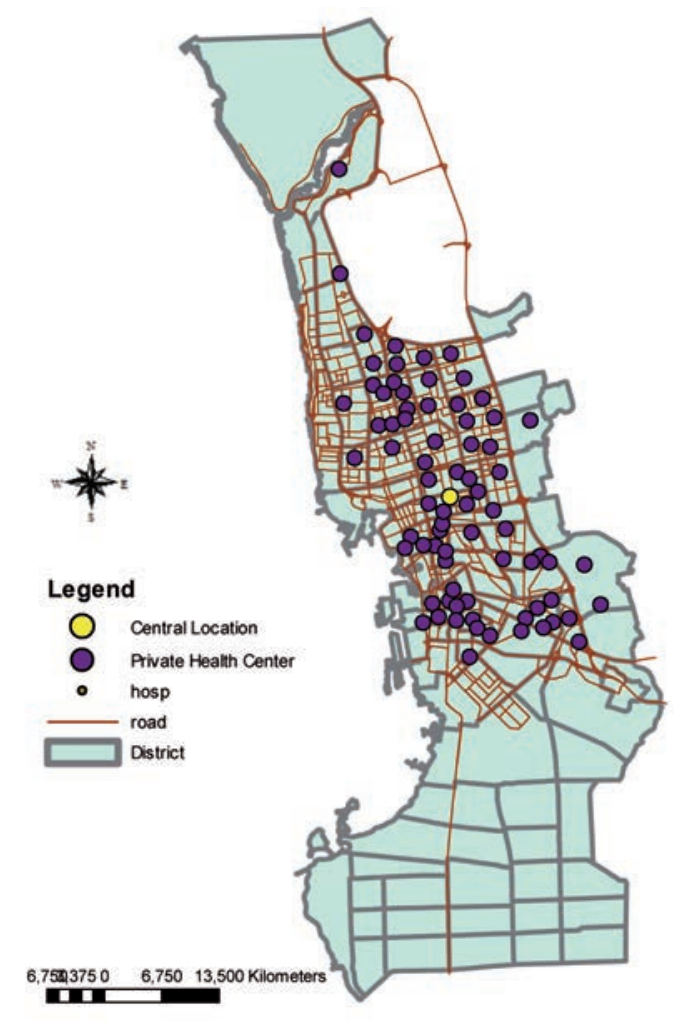

Fig. 10. Spatial distribution of private health centres in Jeddah City.

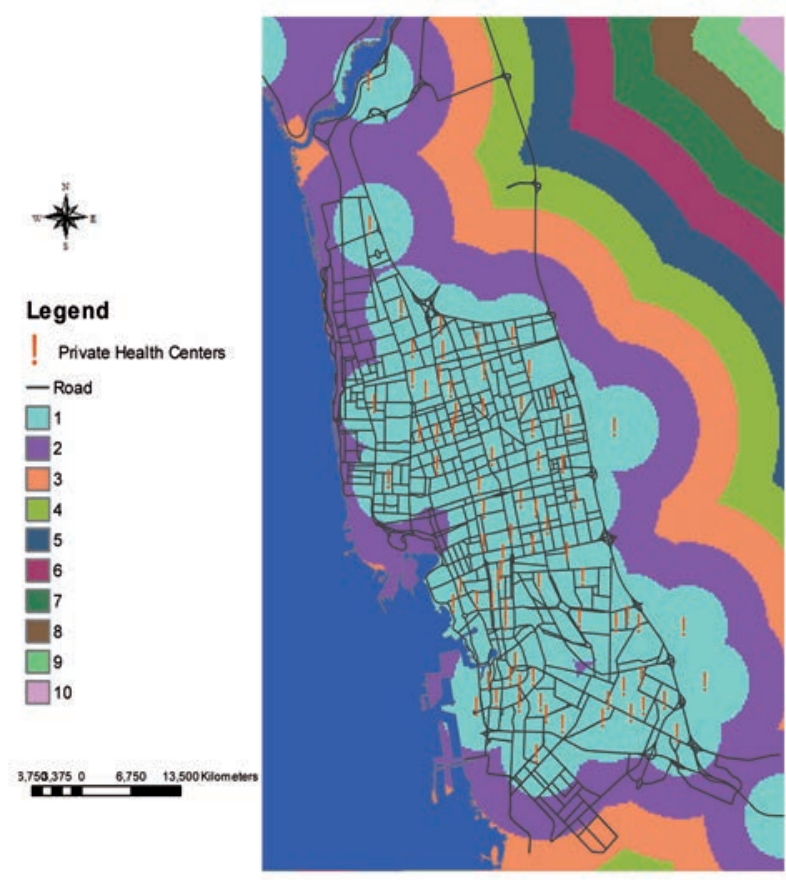

Fig. 11. Accessibility to private health care centres in Jeddah City (based on distance) varies from very accessible (1) to least accessible (12). access to private transportation is limited.

In Saudi Arabia, the private car is considered the main mode of transportation. Therefore, the presented application has produced travel time accessibility zones based on the use of private car. The road network of Jeddah City should allow health service areas to be reached within a 5-min driving-time. However, this model indicates that there are several parts of the city that are located far from the nearest health centre. Accordingly, residents of these areas, who are expected to use private health centres distributed as shown in Fig. 10, have better spatial accessibility (Fig. 11).

The accessibility model based on straight-line distance considers both distance to health centers and the availability of road networks and the results show that there are many parts (mainly in the northern and western districts) of Jeddah City with low health accessibility scores. Health planners need to draw-up future plans for allocations of new public health centres at these zones. The role of geographical accessibility in health service utilisation also depends on population characteristics. People differ in their ability to deal with distance and this influences how locational constraints affect service use. Travel for health care is strongly affected by demographic and socioeconomic characteristics such as income, occupation, age and gender. The presented application is focused on the spatial aspect of health accessibility, but further studies should take into account additional accessibility barriers related to population, time and individuals involved.

Information such as given by the ArcGIS Coverage provides the road network with deatails such as length and type, which is most useful for health planners. It elucidates the spatial pattern of the city network, which subsequently can be overlaid with a health centre location map to visualise the relationship between health facility location and road type. The polygon coverage shows the spatial extent of its health centre for each zone of the city including attributes, such as name of the health centre, population size, patient size, and the extent of the area covered. These data are useful for health planners because they define how large the real catchment area must be and they also define whether centres are sufficiently large with regard to the population size. GIS applied to identify and select features by a special set of criteria with more than one parameter can provide information, such as finding health centres with many physicians but with too few nurses. The features can not only be identified, selected from the database and highlighted on the map according to a combination of several such conditions, but also saved for future reference. 


\section{Conclusions}

GIS technology lends itself well for the evaluation of of the spatial distribution of health services. In the choice between driving-time and the straight-line distance technique, the former turns out to provide a better indication of the geographical barriers to health service, since travel time also incorporates access to transportation. However, walking and/or use of public transportation are especially important in developing countries where access to private transportation is limited. Therefore, several types of spatial data should be collected and health planners need to take all types of transportation into account when planning for relocation or building new public and/or private health facilities. They need also considered the enconomic and social background of the health centres users.

\section{References}

Birkin M, Clarke G, Clarke M, Wilson A,1996. Intelligent GIS: location decisions and strategic planning. Geo information, $292 \mathrm{pp}$.

Braga M, Cislaghi C, Luppi G, Tasco C, 1998. A multipurpose, interactive mortality atlas of Italy. In: GIS and Health. Gathell A, Loytonen M, (eds). London: Taylor and Francis Group, 125-139 pp.

Brown P, Hirschfield A, Batey P, 1991. Applications of geodemographic methods in the analysis of health condition incidence data. Pap Reg Sci 70, 39-44.

Cerrito P, Atnes G, Foibes R, 2003. The analysis of asthma and exposure data using geographic information systems and data mining information. SIAM International Conference on Data Mining. Society for Industrial and Applied Mathematics, 269 273.

Cheng Y, Wang J, Rosenberg M, 2012. Spatial access to residential care resources in Beijing, China. Int J Health Geogr 11, 1-11.

Coggon D, Rose G, Barker D, 1997. Epidemiology for the Uninitiated. BMJ Publishing Group, 113 pp.

Collins S, Smallbone K, Briggs D, 1995. A GIS approach to modeling small area variationions in air pollution within a complex urban environment. In: Innovations in GIS2. Fischer P (ed). London: Taylor and Francis Group, 125-139.

Chou Y, 1997. Exploring spatial analysis in geographic information systems. Onward Press, $97 \mathrm{pp}$.

ESRI, 1992. Network analysis. ESRI, Redlands, CA, USA.

ESRI, 1997. Getting to know Arcview GIS. Geo information. ESRI, Redlands, CA, USA.

ESRI, 2000. GIS in health. ESRI, Redlands, CA, USA.
ESRI, 2001. Getting to know Arc GIS desktop. ESRI, Redlands, CA, USA.

Gatrell A, Loytonen M, 1998. GIS and health research an introduction. In: GIS and Health. Gathell A, Loytonen M (eds). London: Taylor and Francis Group, 14 pp.

Gatrell A, Senior M, 1999. Health and health care applications. In: Geographical information systems. Longley P, Goodchild D, Maguire D, Rhind D (eds). New York: John Wiley and Sons, 925-938 pp.

Jacquez G, 1998. GIS as an enabling technology. In: GIS and Health. Gathell A, Loytonen M (eds). London: Taylor and Francis Group, 187 pp.

Jordan H, Roderick P, Martin D, Barnett S, 2004. Distance, rurality, and the need for care: access to health services in South West England. Int J Health Geogr 3, 1-9.

Jones A, Bentham G, 1995. Emergency medical service accessibility and outcome from road traffic accidents. Public Health 109, 169-77.

Kwang-Soo Lee, Kyeong-Jun Moon, 20014. Hospital distribution in a metropolitan city: assessment by a geographical information system grid modelling approach. Geospat Health 8, 537-544.

Lwasa S, 2007. Geospatial analysis and decision support for health services planning in Uganda. Geospat Health 2, 29-40. Lupien A, Moreland W, Dangermond J, 1987. Network analysis is geographic information system. Photogramm Eng Rem Sens 53, 1417-1421.

Murad A, 2006. Creating a GIS application for health services at Jeddah city. Comput Biol Med 37, 879-889.

Murad A, 2008. Creating a GIS-based epidemiological application for Jeddah city. Int J Healthcare Tech Manag 9, 540-551.

Murad A, 2008. Defining health catchment areas in Jeddah city, Saudi Arabia: an example demonstrating the utility of geographical information systems. Geospat Health 2, 151-160.

Munoz U, Källestål C, 2012. Geographical accessibility and spatial coverage modeling of the primary health care network in the Western Province of Rwanda. Int J Health Geogr 11, 40.

Roovali L, Kiivet R, 2006. Geographical variations in hospital use in Estonia. Health Place 12, 195-202.

Rytkonen M, Moltchanova E, Ranta J, Taskinen O Tuomilehto J, Karbonen M, 2003. The incidence of type 1 diabetes among children in Finland - rural-urban difference. Health Place 9, 315-325.

Wilkinson P, Grundy C, Landon M, Stevenson S, 1998. GIS in Public Health. In: GIS and Health. Gatrell A, Loytonen M (eds). London: Taylor and Francis Group, 179-189 pp.

Zeiler M, 1999. Modeling our world: the ESRI guide to geodatabase design. ESRI, Redlands, CA, USA.

Ranga V, Panda P, 2014. Spatial access to in-patient health care in northern rural India. Geospat Health 8, 545-556. 